\title{
Patients with end-stage kidney disease undergoing hemodialysis: considerations for the dental practitioner
}

\author{
Georges Aoun* \\ Department of Oral Medicine and Maxillofacial Radiology, Faculty of Dental Medicine, Lebanese University, Lebanon
}

\begin{abstract}
Chronic kidney disease is a progressive condition characterized by a gradual loss of kidney functions as result of the nephrons destruction. Patients with end-stage kidney disease (ESKD) require permanent kidney replacement therapy (hemodialysis) to filter toxins and excess fluids from the blood. These patients represent a challenge for the dental practitioner. A sound understanding of ESKD will aid clinicians in safely treating them.
\end{abstract}

\section{Introduction}

The kidneys are bean-shaped organs, located retroperitoneally on the posterior abdominal wall between the transverse processes of the vertebrae T12 and L3. Habitually, in adults, they are $10-12 \mathrm{~cm}$ in length, $5-7 \mathrm{~cm}$ in width, and $3-5 \mathrm{~cm}$ in thickness. Because of the liver, the right kidney is generally positioned slightly inferiorly than the left one [1]. Kidney functions include the excretion of waste products and toxins such as urea, creatinine and uric acid from the blood. Additionally, they control the body's fluid balance and electrolyte level. A nephron is the functional kidney unit consisting of glomerulus, proximal tubules, distal tubules, and collecting duct [2].

Evaluation of kidney function is essential in screening for disorder, monitoring the response to some treatments, and assessing the progression of kidneys diseases. For that, many laboratory tests are used, mainly, the glomerular filtration rate (GFR) and proteinuria (albuminuria) level [2,3]. The international organization "Kidney Disease: Improving Global Outcomes" - KDIGO - guidelines update consensus definitions, staging, and proper laboratory assessment for acute and chronic kidney diseases (CKD) [4]; these guidelines use GFR, commonly considered as the best indicator of kidney function, and albuminuria, a marker of kidney damage, to define and stage kidney diseases [4].

GFR is a laboratory test used to estimate the rate of filtered fluid through the kidney in $\mathrm{ml}$ per minute. Normally, it is about $125 \mathrm{ml}$ per minute per $1.73 \mathrm{~m}^{2}$ and varies according to age, gender, and body size $[3,4]$. A GFR inferior to $60 \mathrm{ml}$ per minute per $1.73 \mathrm{~m}^{2}$ persistent over a period of 3 months is considered resulting from a CKD, with or without absence of kidney damage [5].

\section{Chronic kidney disease}

$\mathrm{CKD}$ is a progressive disease characterized by a gradual loss of kidney functions as result of the nephrons destruction [6,7]. According to the National Institutes of Health, the overall incidence of CKD is approximately $14 \%$, and its main causes are hypertension and diabetes $[2]$.

\section{CKD is classified into 5 stages $[3,6]$ :}

- Stage 1: kidney damage with generally no symptoms and slightly decreased function; GFR is relatively high $\left(\geq 90 \mathrm{ml} / \mathrm{min} / 1.73 \mathrm{~m}^{2}\right)$ to normal.

- Stage 2: kidney damage with generally no symptoms and mild decrease in GFR $\left(60-89 \mathrm{ml} / \mathrm{min} / 1.73 \mathrm{~m}^{2}\right)$.

- Stage 3: kidney damage with moderate decrease in GFR (30-59 $\mathrm{ml} / \mathrm{min} / 1.73 \mathrm{~m}^{2}$ ). At this stage, levels of urea increase in the blood (uremia), and the patient is likely to develop complications of kidney disease such as high blood pressure, anemia etc.

- Stage 4: advanced kidney damage with severe decrease in GFR $\left(15-30 \mathrm{ml} / \mathrm{min} / 1.73 \mathrm{~m}^{2}\right)$. At this stage, the patient is at higher risk of developing severe complications of kidney disease.

- Stage 5: end-stage kidney disease (ESKD) with GFR $<15 \mathrm{ml} /$ $\min / 1.73 \mathrm{~m}^{2}$.

While the treatment in the early stages of the disease aims to slow down the progression of the kidney damage habitually by controlling the underlying cause, the ESKD, which is a lethal condition, requires kidney transplantation or, if not available or possible, permanent kidney replacement therapy (hemodialysis) to filter toxins and excess fluids from the blood using a dialyzer considered as an artificial kidney [4,8].

\section{Chronic kidney disease and hemodialysis}

Progression of CKD is linked to many severe complications, including anemia, dyslipidemia, cardiovascular and metabolic bone diseases [9].

"Correspondence to: Professor Georges Aoun, Department of Oral Medicine and Maxillofacial Radiology, Faculty of Dental Medicine, Lebanese University, Beirut, Lebanon, ORCID ID: http://orcid.org/0000-0001-5073-6882, E-mail: dr.georgesaoun@gmail.com

Key words: dentist, hemodialysis, kidney failure, management, oral manifestations Received: June 14, 2021; Accepted: July 08, 2021; Published: July 12, 2021 
Anemia related to CKD mostly results from the reduction of erythropoietin secretion by the kidney interstitial fibroblasts as a consequence of tubular atrophy and tubulointerstitial fibrosis $[9,10]$. Erythropoietin is indispensable for red blood cell growth and differentiation in the bone marrow [9].

Dyslipidemia, which is largely linked to CKD, is an important risk factor for cardiovascular diseases. In fact, hyperlipidemia increases as kidney function decreases, with hypertriglyceridemia and elevation of LDL cholesterol being proportional to the severity of the kidney's impairment $[9,11]$. Hypercholesterolemia results from increased production of lipoproteins and their decreased catabolism [9].

The high risk of cardiovascular diseases related to CKD has been well-established. In fact, even mild to moderate levels of kidney impairment increases such risk [12]. Among others, hypertension mainly contributes to the cardiovascular risk connected with CKD [13]. Moreover, anemia of CKD worsens cardiovascular complications such as angina, left ventricular hypertrophy and heart failure [14], ultimately causing additional deterioration of kidney function and hence leading to a vicious cycle known as the "cardio-renal anemia syndrome" $[9,14]$.

Additionally, CKD may lead to mineral and bone disorders and/or extra-skeletal calcification [15]. Patients with CKD develop hyperphosphatemia as result of the decrease of renal phosphate excretion and hypocalcemia because of the cessation of calcitriol (the active form of vitamin D) activation by the kidneys. Consequently, parathyroid glands release parathyroid hormone into the blood to raise calcium levels by increasing bone resorption. This response restores phosphorus and calcium balance; however, it also deprives bone of calcium leading to changes in bone architecture $[9,15]$. This disorder in calcium, phosphorus, and vitamin $\mathrm{D}$ metabolism and increase in parathyroid activity is known as renal osteodystrophy [6]. As for the extra-skeletal calcification, it is believed to be linked to hyperparathyroidism and vascular calcification, which results from high phosphorus levels $[16,17]$.

With the progression of CKD toward ESKD, the severity of the complications increases and worsens requiring aggressive measures such as dialysis to be taken. Hemodialysis consists in eliminating nitrogenous and other toxic products from the blood using an artificial kidney containing semipermeable membranes that allow the passage of excess fluid and wastes. Access to the bloodstream is secured through arteriovenous shunts or fistulas. During the procedure, patients are given anticoagulants to facilitate blood circulation through the dialysis membrane [8]. Usually, patients undergo 3 sessions of hemodialysis per week, each one for 3 to 4 hours [7,9].

\section{Oral manifestations in patients with end-stage kidney disease undergoing hemodialysis}

Oral manifestations in patients with ESKD undergoing hemodialysis may include: 1) pallor of the mucosa, 2) risk of bleeding, 3) predisposition to ecchymoses, petechiae, and hemorrhage, 4) xerostomia, 5) uremic halitosis and metallic taste, 6) alteration of flavors especially sweet and acid, 7) mouth burning and enlarged tongue sensations, 8) uremic stomatitis, 9) lichenoid lesions, 10) periodontitis, 11) advanced periodontal disease (deep pockets, bone resorption, 12) tooth mobility and malocclusion, 13) pulp stones,14) enamel hypoplasia, 15) bone demineralization, 16) decreased trabeculation of cancellous bone and thickness of cortical bone, 17) giant cell lesions, 18) abnormal bone healing and risk of jaw fracture (spontaneous or after dental extractions).

These oral manifestations and their probable etiologies are summarized in Table 1 .

\section{Risks and management in the dental office}

Patients with ESKD undergoing hemodialysis require special consideration. The communication with the nephrologist is mandatory in order to better comprehend the severity of the case, medications intake, and presence of other diseases (cardiovascular diseases, diabetes, etc.) that may negatively affect the patient's condition [23]. Other significant risks taken into consideration include the risk of bleeding and the increased susceptibility to infection [6].

Dental procedures with risk of bleeding should be carefully planned. Anemia, alteration in platelet aggregation, and anticoagulant effect of heparin administrated the day of dialysis increase the bleeding risk [6]. For that, any risky dental treatment should be delayed to a non-dialysis day to ensure that the patient is not exhausted after a long dialysis session, as well as to have their bloodstream free from the effect of heparin and toxic metabolites. Additionally, hematologic tests such

Table 1. Oral manifestations in patients with ESKD undergoing hemodialysis and their etiologies

\begin{tabular}{|c|c|}
\hline Oral Manifestation & Etiology \\
\hline Pallor of the mucosa & Anemia $[18]$ \\
\hline Risk of bleeding & Platelet aggregation problem and anemia $[18,19]$ \\
\hline Predisposition to ecchymoses, petechiae, and hemorrhage & Platelet dysfunction, renal anemia, and the use of anticoagulants [20] \\
\hline Xerostomia & Patient discouraged from drinking much fluids $[6,20]$ \\
\hline Uremic halitosis and metallic taste & High urea concentration in saliva [7] \\
\hline Alteration of sweet and acidic flavors & High urea concentration in saliva [6] \\
\hline Burning mouth sensation & Neuropathic [19] \\
\hline Uremic stomatitis & Unknown but some researchers incriminate uremia [7] \\
\hline Lichenoid lesions & Antihypertensive medication [21] \\
\hline Periodontitis & Poor hygiene and/or diabetic nephropathic $[18,22]$ \\
\hline Advanced periodontal disease (deep pockets, bone resorption...) & Renal osteodystrophy [18] \\
\hline Tooth mobility and malocclusion & Renal osteodystrophy $[7,20]$ \\
\hline Pulp stones & Renal osteodystrophy $[7,20]$ \\
\hline Enamel hypoplasia & Renal osteodystrophy $[7,20]$ \\
\hline Decreased trabeculation of cancellous bone and thickness of cortical bone & Renal osteodystrophy $[7,20]$ \\
\hline Giant cell lesions & Renal osteodystrophy $[7,20]$ \\
\hline Risk of jaw fracture and abnormal bone healing & Renal osteodystrophy $[7,20]$ \\
\hline
\end{tabular}


as platelets count, hematocrit, and hemoglobin should be done prior to such procedures [6].

Furthermore, in patients on warfarin, the International Normalized Ratio (INR) should be tested; it is believed that minor surgeries can be safely done with INR ranging between 2.5 and 4 [5,24]. In case of necessity, the administration of protamine sulfate to counteract the effect of heparin is recommended. Moreover, postoperatively, local hemostatic measures can be utilized to stop localized bleeding [6] Nevertheless, new oral anticoagulants require more careful evaluation and communication with the treating physician as these cannot be evaluated using INR as warfarin.

Dental local anesthesia can be safely administered because of the hepatic elimination of the anesthetics. As for the medications, paracetamol remains the best analgesic of choice; on the other hand, nonsteroidal anti-inflammatory drugs should be avoided for their adverse effects on blood pressure and their potential to increase kidney injury in patients with CKD. Furthermore, aspirin is contraindicated because of its antiplatelet effect [6]. Additionally, to avoid adrenal crisis, dentists should discuss with the treating physician the necessity of steroid supplementation in patients who have been treated with longterm high doses glucocorticoids and apply a stress-free protocol in pre-, intra- and post-dental treatment [23].

Finally, ESKD patients undergoing hemodialysis can develop bacteraemia following the infection of the vascular access thus needing antibiotic prophylaxis to prevent infective endocarditis. For that, vancomycin infusion during dialysis is recommended [25]. On the other hand, in the presence of a dental infection, a full course of a nonnephrotoxic antibiotic should be administered.

\section{Conclusion}

$\mathrm{CKD}$ is a global progressive disease characterized by a gradual loss of kidney functions. ESKD requires kidney replacement therapy through hemodialysis or kidney transplant. In the presence of an ESKD patient, dentists should coordinate with the treating nephrologist the dental procedure in order to avoid possible complications during and/ or after the dental treatment. Additionally, they should motivate the patient for oral hygiene, try to detect ESKD oral manifestations early and take adequate preventive measures.

\section{References}

1. El-Reshaid W, Abdul-Fattah H (2014) Sonographic assessment of renal size in healthy adults. Med Princ Pract 23: 432-436. [Crossref]

2. Gounden V, Bhatt H, Jialal I (2020) Renal Function Tests. In: StatPearls [Internet]. Treasure Island (FL). [Crossref]

3. Kaufman DP, Basit H, Knohl SJ (2021) Physiology, Glomerular Filtration Rate. In: StatPearls [Internet]. Treasure Island (FL). [Crossref]
4. Stevens PE, Levin A, Kidney Disease: Improving Global Outcomes Chronic Kidney Disease Guideline Development Work Group Members (2013) Evaluation and management of chronic kidney disease: synopsis of the kidney disease: improving global outcomes 2012 clinical practice guideline. Ann Intern Med 158: 825-830. [Crossref]

5. Georgakopoulou EA, Achtari MD, Afentoulide N (2011) Dental management of patients before and after renal transplantation. Stomatologija 13: 107-112. [Crossref]

6. Costantinides F, Castronovo G, Vettori E, Frattini C, Artero ML, et al. (2018) Dental Care for Patients with End-Stage Renal Disease and Undergoing Hemodialysis. Int $J$ Dent 2018: 9610892.

7. De Rossi SS, Glick M (1996) Dental considerations for the patient with renal disease receiving hemodialysis. J Am Dent Assoc 127: 211-219. [Crossref]

8. Vasanthan A, Dallal N (2007) Periodontal treatment considerations for cell transplant and organ transplant patients. Periodontol 2000 44: 82-102. [Crossref]

9. Thomas R, Kanso A, Sedor JR (2008) Chronic kidney disease and its complications. Prim Care 35: 329-344. [Crossref]

10. Ratcliffe PJ (1993) Molecular biology of erythropoietin. Kidney Int 44: 887-904. [Crossref]

11. Kasiske BL (1998) Hyperlipidemia in patients with chronic renal disease. Am J Kidney Dis 32: S142-156. [Crossref]

12. Foley RN, Parfrey PS, Sarnak MJ (1998) Clinical epidemiology of cardiovascular disease in chronic renal disease. Am J Kidney Dis 32: S112-119. [Crossref]

13. Muntner P, He J, Astor BC, Folsom AR, Coresh J (2005) Traditional and nontraditional risk factors predict coronary heart disease in chronic kidney disease: results from the atherosclerosis risk in communities study. J Am Soc Nephrol 16: 529-538. [Crossref]

14. Besarab A, Levin A (2000) Defining a renal anemia management period. Am J Kidney Dis 36: S13-S23. [Crossref]

15. Gal-Moscovici A, Sprague SM (2007) Bone health in chronic kidney disease-mineral and bone disease. Adv Chronic Kidney Dis 14: 27-36. [Crossref]

16. El-Kishawi AM, El-Nahas AM (2006) Renal osteodystrophy: review of the disease and its treatment. Saudi J Kidney Dis Transpl 17: 373-382. [Crossref]

17. Hutchison JA (2007) Vascular calcification in dialysis patients. Prilozi 28: 215-224. [Crossref]

18. Klassen JT, Krasko BM (2002) The dental health status of dialysis patients. J Can Dent Assoc 68: 34-38. [Crossref]

19. Leão JC, Gueiros LA, Segundo AV, Carvalho AA, Barrett W, eT al. (2005) Uremic stomatitis in chronic renal failure. Clinics (Sao Paulo) 60: 259-262. [Crossref]

20. Dioguardi M, Caloro GA, Troiano G, Giannatempo G, Laino L, et al. (2016) Oral manifestations in chronic uremia patients. Ren Fail 38: 1-6. [Crossref]

21. Proctor R, Kumar N, Stein A, Moles D, Porter S (2005) Oral and dental aspects of chronic renal failure. J Dent Res 84: 199-208. [Crossref]

22. Nylund K, Meurman JH, Heikkinen AM, Honkanen E, Vesterinen M, et al. (2015) Oral health in predialysis patients with emphasis on periodontal disease. Quintessence Int 46: 899-907. [Crossref]

23. Martí-Álamo S, Gavaldá-Esteve C, Sarrión-Pérez MG (2011) Dental considerations for the patient with renal disease. J Clin Exp Dent 3: e112-e119. [Crossref]

24. Saif I, Adkins A, Kewley V, Woywodt A, Brookes V (2011) Routine and emergency management guidelines for the dental patient with renal disease and kidney transplant. Part 1. Dent Update 38: 179-182, 185-186. [Crossref]

25. Tobin M, Montes M, Mookerjee BK (1978) Endocarditis in hemodialysis patients with systemic disease. J Dial 2: 75-84. [Crossref]

Copyright: (C2021 Aoun G. This is an open-access article distributed under the terms of the Creative Commons Attribution License, which permits unrestricted use, distribution, and reproduction in any medium, provided the original author and source are credited. 